\title{
Validation of the repetitive and restricted behaviour scale in autism spectrum disorders
}

\author{
Yannig Bourreau $\cdot$ Sylvie Roux $\cdot$ Marie Gomot $\cdot$ \\ Frédérique Bonnet-Brilhault · Catherine Barthélémy
}

Received: 6 August 2008/Accepted: 29 April 2009/Published online: 19 May 2009

(c) Springer-Verlag 2009

\begin{abstract}
Repetitive and restricted behaviours represent a common problem for various psychiatric syndromes, especially in autistic spectrum disorders, and they include a wide range of heterogeneous behavioural manifestations. An accurate and standardized description of these behaviours is needed to advance the understanding of this complex and heterogeneous clinical dimension of autism. The present article reports the reliability and validity studies of a new assessment scale: the repetitive and restricted behaviour scale. 145 subjects with autism spectrum disorders were assessed using the RRB scale. The RRB scale has good interrater reliability, internal consistency and content validity. Factorial analysis produced four clinically meaningful factors, i.e. "sensorimotor stereotypies", "reaction to change", "restricted behaviours" and "modulation insufficiency". The RRB scale has good psychometric qualities and constitutes a real breakthrough towards a neurofunctional approach to autistic disorders. It should be valuable for research and treatment, and in clinical practice.
\end{abstract}

Keywords Autism spectrum disorder .

Stereotyped behaviour $\cdot$ Assessment scale $\cdot$ Validation

Y. Bourreau $\cdot$ S. Roux $\cdot$ M. Gomot · F. Bonnet-Brilhault .

C. Barthélémy

UMRS Inserm U930, CNRS ERL3106, Université François

Rabelais de Tours, CHRU de Tours, Tours, France

S. Roux $(\bowtie)$

Laboratoire d'Explorations Fonctionnelles et Neurophysiologie en Pédopsychiatrie, CHRU de Tours,

37044 Tours cedex 09, France

e-mail: s.roux@chu-tours.fr

\section{Introduction}

Repetitive behaviours, stereotyped activities and restricted interests, summarized as repetitive and restricted behaviours (RRB), can occur in a number of psychiatric disorders, particularly in autism spectrum disorders (ASD), obsessive compulsive disorders (OCD), mental retardation, and Tics and Tourette syndrome [6, 13, 23]. In ASD, RRB constitute the third dimension of diagnostic criteria $[1,45]$ which covers a wide range of heterogeneous behavioural manifestations such as motor stereotypies, sensory-related behaviours, circumscribed interests, rituals, excessive sensitivity to change, echolalia, and selfinjurious behaviours $[10,13,23,25,39,40]$. Their physiopathological mechanisms still remain unsolved $[10,13,23,25,40]$. All these behaviours point to a lack of flexibility that results in major difficulties in daily life, both for the individual and his or her relatives [16, 25, 26]. Most of the previous research has divided RRB into low-level and high-level behaviours $[4,7,10,13,17,27$, 30, 39, 40]. Low-level RRB correspond to repetitive sensorimotor behaviours and are said to be linked to younger age and associated with mental retardation $[4,10$, 25]. Conversely, high-level RRB refer to more complex repetitive activities (rituals) and insistence on sameness. They seem to develop with age and to be more strongly expressed in high-functioning individuals [4, 17, 39]. High-level RRB might also be more specific of autism than sensorimotor RRB [10, 40].

However, studies dealing with RRB in ASD to date have not taken into account all the forms of RRB and have rarely used specific tools.

The repetitive behaviour scale (RBS and its revised version the RBS-R) has been the most complete and the only specific tool for the assessment of RRB in autism 
$[5,6]$. The RBS-R is based on a descriptive approach to the behaviours observed and was recently re-validated [21]. It contains 43 items but lacks some aspects of RRB, such as echolalia, mannerisms and stereotyped emotional manifestations.

We have developed a new assessment scale based not only on a purely descriptive evaluation, but also on a neurofunctional approach to behaviours, i.e. the RRB scale. It covers the whole range of repetitive and restricted behaviours observed in ASD. This new scale should allow first the definition of more objective and homogeneous subsets of RRB that might be related to different psychophysiological mechanisms, and then the improvement of therapeutic strategies and evaluation of their efficacy. The present article reports the reliability and validity studies of the RRB scale.

\section{Methods}

\section{The RRB scale}

The construction of the RRB scale was based on clinical observations and on an extensive review of the literature on RRB in both autism and mental retardation $[2,5,9,20,21$, $23-25,33,34,36,40]$. An initial list of 43 items, corresponding to the most frequent or characteristic RRB was tested on a small sample of children with ASD. This list gave a satisfactory outline of RRB and confirmed their heterogeneity [7]. This list was then submitted to clinical experts (child psychiatrists, psychologists, speech therapists, nurses) in order to make it more accurate and comprehensible, and thus to confirm its face validity.

The present version of the RRB scale comprises 35 items (items detailed in Table 2) explained in a glossary (available on request) and evaluated according to a fivelevel Likert scale $(0=$ "the behaviour is never expressed by the person", 1 = "weakly expressed", $2=$ "moderately expressed", $3=$ "severely expressed" and $4=$ "the behaviour is very characteristic of the person and very severely expressed"). Since RRB constitute a complex and heterogeneous dimension, a sound understanding of the person is necessary to complete the scale with accuracy. The RRB scale is therefore filled out by professional caregivers after they have observed the person in various situations of daily life (during free and structured activities, alone and in a group, during mealtimes...). Since some behaviours, such as restricted interests and resistance to change, can be difficult to rate, direct observation can be complemented by information collected from a relative of the subject. Moreover, the rater does not have to take into account RRB that were typical of the person but are no longer observed.
Population

Validation of the RRB scale required a large and varied population in terms of age, intellectual capacities, symptom severity, and residential settings. Thirteen centres took part in the validation study (for details see "Acknowledgments"). Informed consent was obtained from the parents, and anonymous data were collected and analysed in an INSERM research centre authorized by the Direction Générale de la Santé (No 06032).

The participants were 145 children, adolescents and adults ( 38 female, 107 male) aged from 3 to 33 years: there were 49 young children (aged from 3 to 7 years), 40 children (8-12 years), 32 teenagers (13-18 years), and 24 adults (19-33 years).

Disorders were diagnosed by expert clinicians according to DSM-IV-R criteria [1] as follows: autistic disorder (AD, $n=99$ ), pervasive developmental disorder-not otherwise specified (PDD-NOS, $n=41$ ) and Asperger Syndrome (AS, $n=5$ ). The ADI-R [24] was collected for $32 \%$ of the sample to confirm the diagnoses. No major neurological syndromes were diagnosed. Intellectual abilities [development quotient (DQ) or intellectual quotient (IQ)] was evaluated with different tests depending on age and ability and on centre (BL-R, EDEI-R, WISC-III, WISC-IV, PEP-R, WPPSI-III, Nemi, and Leiter-R [8, 28, 32, 35, 41-43, 47]). According to DSM-IV-R criteria, 24 subjects had profound mental retardation, 33 had severe mental retardation, 37 had moderate mental retardation, 25 had slight mental retardation, and 26 had no mental retardation.

The severity of autistic symptoms was assessed with the CARS [29, 36]; data were available for 121 subjects.

Participants' characteristics are presented in Table 1.

The ages of males and females were similar. Males had higher DQ-IQ than females $(t=2.53, d f=143$, $p=0.012)$; however, the difference was no longer significant when considering only participants with DQ-IQ below 80. Individuals with $\mathrm{AD}$ and PDD-NOS did not differ in age or DQ-IQ.

\section{Subpopulation to assess interrater reliability}

A subgroup of 21 children and 8 adults ( 7 female, 22 male), aged 3-33 years $(M=12, \mathrm{SD}=9)$ was assessed for

Table 1 Participants' characteristics

\begin{tabular}{llrccc}
\hline & $\begin{array}{l}\text { Number of } \\
\text { subjects }\end{array}$ & Mean & $\begin{array}{l}\text { Standard } \\
\text { deviation }\end{array}$ & Minimum & Maximum \\
\hline Age (years) & 145 & 12.2 & 7.3 & 3 & 33 \\
DQ-IQ & 145 & 45.0 & 25.4 & 6 & 126 \\
CARS & 121 & 34.9 & 7.0 & 20 & 55.5 \\
Covi & 88 & 7.1 & 2.7 & 3 & 14 \\
\hline
\end{tabular}


interrater reliability. The children were individuals from the Child Psychiatry Day Unit of the "Centre Hospitalier Universitaire" in Tours and the adults were residents of "les Maisonnées", a centre at Azay le Rideau. Diagnoses in this subpopulation were $\mathrm{AD}(n=16)$ and PDD-NOS $(n=13)$. Overall DQ-IQ ranged from 10 to 114 $(M=55.0 ; \mathrm{SD}=23.9)$.

Procedures

\section{Validity study}

The factor structure of the RRB scale was explored using principal component analysis (PCA) with varimax rotation. The number of factors to be retained was defined by the scree-test criterion and complemented by screeplots of simulated random data. Items were included in the factor structure if they loaded 10.35 I or higher on a factor, and if the loading was at least 10.10 l higher than the loading on any other factor. All the items loading highly on a factor were referred to as a subscale. They were combined to produce an average score for each subscale. The correlations between each item and the overall score of each subscale were computed to confirm the factor structure. Finally, the Cronbach $\alpha$ coefficient was used to assess internal consistency of each subscale.

Relationships between subscale scores and participant characteristics (i.e. age, DQ-IQ, severity of autistic symptoms (measured with the CARS) and anxiety) were assessed using Pearson's product moment correlation. Anxiety is not a core feature of ASD, but it can explain the expression of RRB [38, 40]. We, therefore, used the Covi anxiety scale which contains three items rated on a fivepoint scale and provides easy and rapid assessment of anxiety based on observation of the individual [12, 22, 31] (see Table 1). Standard multiple regression analyses were then performed to complement the interpretation of the relationships between the variables (subscale score as dependent variable).

Additional statistical analyses were also carried out to complement the main results $\left(t\right.$ tests, $\chi^{2}$ tests, and ANOVA with Newman-Keuls post hoc tests). STATISTICA v8 (StatSoft, Inc.) was used.

\section{Interrater reliability study}

To check the reliability of the RRB scale, two raters independently filled out the RRB scale for each subject, according to the above-mentioned principles. Reliability was then ascertained by calculating the weighted kappa statistic (Kw) for each of the 35 items $[11,14,15]$ and the intraclass correlation coefficient (ICC) on the score of each subscale.

\section{Results}

Validity study

\section{Factor structure}

Frequency of occurrence of each behaviour was calculated on the basis of present (ratings 1,2,3, and 4) versus absent (rating 0) scores. All items occurred in more than $10 \%$ and less than $90 \%$ of the sample. The 35 items were thus retained for subsequent analysis.

PCA was performed on the 145 subjects and produced four factors that cumulatively accounted for $43 \%$ of total variance (screeplots are presented on Fig. 1 and factor loadings are reported in Table 2). These four factors were fairly similar in terms of number of items $(11,7,8$, and 7 , respectively) and percentage of explained variance $(12,11$, 11 , and 9 , respectively).

Factor 1 (F1) comprised 11 items corresponding to repetitive motor behaviours, motor mannerisms, repetitive cries, body-focused behaviours and atypical sensory behaviours. This factor was labelled "sensorimotor stereotypies". Factor 2 (F2) comprised seven items that reflected adoption of rituals for various activities, reaction to small changes in the environment and echolalia. It was labelled "reaction to change". Factor 3 (F3) comprised eight items dealing with repetitive or ritualized use of objects, interest in or attachment to objects or details of objects, circumscribed subjects of interest and lack of interest in novelty. It was labelled "restricted behaviours". Factor 4 (F4) comprised seven items that included aggressiveness towards self and others, need to control the progress of activities, stereotyped emotional manifestations and agitation. This factor was labelled "modulation insufficiency".

All items of the RRB scale correlated more highly with their subscale (from 0.59 to 0.66 ) than with the other subscales (less than 0.21). The correlations between the four factors ranged from 0.05 to 0.32 . The Cronbach $\alpha$ coefficient showed good internal consistency for each subscale $(0.81,0.79,0.75$, and 0.72 , respectively).

No difference was found between males and females for the four subscale scores. In terms of diagnosis, subjects with AD had higher scores than those with PDD-NOS on three subscale scores: $\mathrm{F} 1(t=2.79, d f=138, p=0.006)$, $\mathrm{F} 2(t=3.36, d f=138, p<0.001)$, and F3 $(t=4.86$, $d f=138, p<0.001)$.

Significant negative correlations were found between F1 and F3 scores and DQ-IQ. Since CARS score has been known to be linked to level of mental retardation [25] (in this study, $r=0.51, p<0.001)$, partial correlations were computed between subscale scores and CARS score: F1, F3, and F4 scores showed significant correlations. For the 
Fig. 1 Screeplots produced by PCA of the RRB scale and of ten simulated random datasets

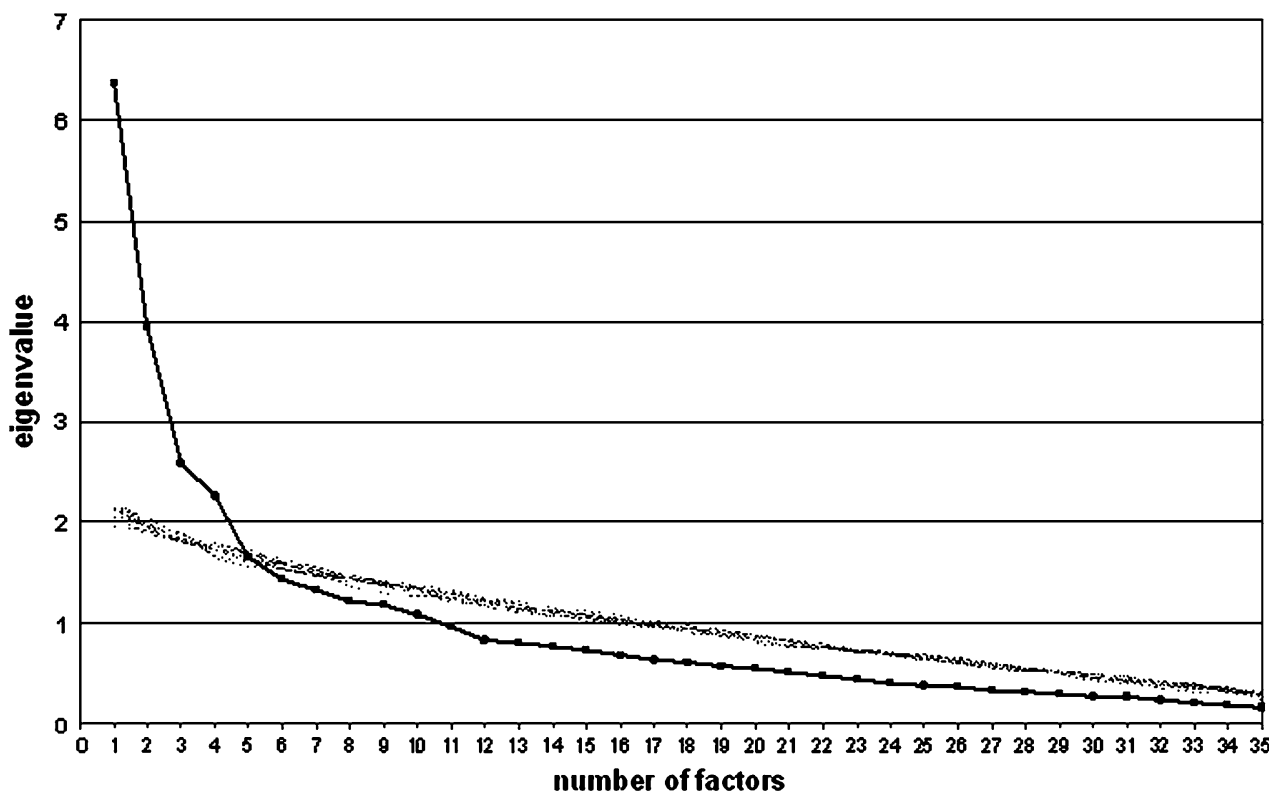

Dotted lines: simulated random data
Covi score, a slight negative correlation was found with the F3 score. Conversely, F4 was positively correlated with anxiety (see Table 3 ).

Standard multiple regression analyses were then performed to clarify the relationships previously shown between F1, F3 and F4 subscale scores on the one hand and CARS score, DQ-IQ, and Covi score on the other. These analyses made it possible to examine the relative importance of these three independent variables in the explanation of F1, F3, and F4 subscale scores. Only the CARS score made a statistically significant contribution to the explanation of the F1 score $\left[R^{2}=0.44\right.$, adjusted $R^{2}=$ $0.42, F(3,76)=20.27, p<0.001]$. All three independent variables contributed statistically to the explanation of the F3 score $\left[R^{2}=0.29\right.$, adjusted $R^{2}=0.26, F(3,76)=10.37$, $p<0.001]$. Finally, CARS and Covi scores made a statistically significant contribution to the explanation of the F4 score $\left[R^{2}=0.26\right.$, adjusted $R^{2}=0.23, F(3,76)=8.87$, $p<0.001]$, and the Covi score made the greatest contribution (see Table 4).

\section{Interrater reliability study}

\section{The RRB scale showed good reliability}

Using the criteria defined by Cicchetti [11], 9 items (items $1,6,16,18,22,29,30,32,34)$ had excellent $(\mathrm{Kw}=0.75-$ 1) reliability, 17 items (items $2,3,5,7,8,9,11,13,17,19$, $21,23,24,25,26,31,33)$ had good (0.60-0.74) reliability and 9 items (items 4, 10, 12, 14, 15, 20, 27, 28, 35) had moderate (0.40-0.59) reliability (see Table 2 ).
The ICC values for the subscales were 0.88 (95\% confidence interval: 0.76-0.94) for "sensorimotor stereotypies" (F1), $0.87(0.74-0.94)$ for "reaction to change" (F2), 0.84 (0.68-0.92) for "restricted behaviours" (F3), and 0.94 (0.87-0.97) for "modulation insufficiency" (F4).

\section{Discussion}

The validation study showed the sound psychometric qualities of the RRB scale and its ability to describe four clinically meaningful components within the repetitive and restricted behaviours of ASD, i.e. sensorimotor stereotypies, reaction to change, restricted behaviours, and modulation insufficiency. Of these four factors, three were more severely expressed in AD compared to PDD-NOS. Moreover, sensorimotor stereotypies and reaction to change were not influenced by level of mental retardation or by anxiety, and they remained stable over time. These two dimensions, therefore, appear to be integral parts of autistic core symptoms. On the other hand, restricted behaviours, which were particularly related to level of mental retardation, and modulation insufficiency, mainly linked to the expression of anxiety, are probably less specific of ASD.

Features and content of the RRB scale

The four dimensions of the RRB scale did not appear to be influenced by age. This is consistent with studies emphasising the persistence with age of this dimension of autistic disorder [18, 37]. 
Table 2 Factor loadings and weighted kappa statistic Kw of the 35 items of the RRB scale

\begin{tabular}{|c|c|c|c|c|c|c|}
\hline & \multirow[t]{2}{*}{ Item } & \multirow[t]{2}{*}{$\mathrm{Kw}$} & \multicolumn{4}{|c|}{ Factor loadings ( $\%$ of variance) } \\
\hline & & & F1 $(12 \%)$ & $\mathrm{F} 2(11 \%)$ & F3 $(11 \%)$ & F4 $(9 \%)$ \\
\hline 1 & Repetitive body rocking & 0.76 & 0.65 & 0.09 & 0.06 & -0.01 \\
\hline 2 & Repetitive and atypical movements of mouth & 0.64 & 0.61 & 0.02 & -0.09 & 0.05 \\
\hline 3 & Repetitive movements of head & 0.70 & 0.67 & 0.08 & 0.14 & -0.12 \\
\hline 4 & Repetitive movements of arms and hands & 0.56 & 0.56 & -0.18 & 0.14 & 0.08 \\
\hline 5 & Bizarre gait & 0.64 & 0.53 & 0.10 & -0.11 & 0.09 \\
\hline 6 & Repetitive pacing & 0.75 & 0.31 & 0.03 & 0.40 & 0.40 \\
\hline 7 & Mannerism or rigidity of posture & 0.69 & 0.59 & 0.20 & -0.17 & 0.01 \\
\hline 8 & $\begin{array}{l}\text { Quick and complex movements of fingers in front } \\
\text { of eyes, with or without objects }\end{array}$ & 0.67 & 0.59 & -0.20 & 0.37 & 0.12 \\
\hline 9 & Repetitive and non-functional use of objects & 0.65 & 0.34 & -0.05 & 0.66 & -0.01 \\
\hline 10 & Interest in a detail of objects & 0.46 & 0.36 & 0.21 & 0.60 & 0.06 \\
\hline 11 & Attachment to certain objects & 0.64 & -0.03 & 0.15 & 0.63 & 0.23 \\
\hline 12 & Circumscribed interests & 0.57 & -0.04 & 0.26 & 0.50 & 0.09 \\
\hline 13 & Exact repetition of words, sentences or tunes & 0.72 & -0.03 & 0.53 & -0.24 & -0.03 \\
\hline 14 & Repetitive uttering of cries or sounds & 0.56 & 0.54 & -0.05 & 0.27 & 0.26 \\
\hline 15 & Aggressive behaviours towards others or objects & 0.51 & -0.01 & -0.06 & 0.07 & 0.71 \\
\hline 16 & Self-injurious behaviours & 0.75 & 0.28 & -0.08 & 0.21 & 0.48 \\
\hline 17 & Body-focused behaviours & 0.73 & 0.51 & -0.22 & 0.07 & 0.30 \\
\hline 18 & Rituals for daily living activities & 0.76 & 0.03 & 0.69 & 0.17 & 0.13 \\
\hline 19 & Play and leisure rituals & 0.73 & 0.02 & 0.68 & 0.41 & -0.15 \\
\hline 20 & Communication rituals & 0.55 & 0.05 & 0.67 & -0.06 & 0.16 \\
\hline 21 & Route and exploration rituals & 0.74 & 0.06 & 0.62 & 0.27 & 0.16 \\
\hline 22 & Alignment rituals & 0.75 & -0.14 & 0.24 & 0.58 & 0.17 \\
\hline 23 & Reaction when progress of a ritual or activity is disturbed & 0.61 & 0.07 & 0.44 & 0.29 & 0.52 \\
\hline 24 & Repetitive tendency to hoard objects & 0.63 & -0.15 & -0.11 & 0.58 & 0.29 \\
\hline 25 & Tendency to try to control activities or conversation & 0.66 & -0.20 & 0.38 & -0.15 & 0.60 \\
\hline 26 & Atypical sensory behaviours & 0.72 & 0.53 & 0.12 & 0.12 & 0.29 \\
\hline 27 & Attraction to things that move & 0.56 & 0.15 & -0.15 & 0.59 & -0.04 \\
\hline 28 & Attraction to certain sounds or noises & 0.55 & 0.42 & -0.02 & 0.28 & 0.10 \\
\hline 29 & Interest in a part of the body of others & 0.90 & 0.33 & 0.01 & -0.14 & 0.47 \\
\hline 30 & Need that things be laid out in a specific pattern & 0.79 & 0.03 & 0.45 & 0.48 & 0.00 \\
\hline 31 & Reaction to changes in material environment & 0.65 & -0.04 & 0.65 & 0.29 & -0.16 \\
\hline 32 & Reaction to changes in appearance or behaviours of relatives & 0.85 & 0.06 & 0.61 & -0.05 & 0.09 \\
\hline 33 & Lack of interest in novelty & 0.60 & 0.08 & 0.07 & 0.37 & -0.11 \\
\hline 34 & Stereotyped emotional manifestations & 0.77 & 0.17 & 0.20 & 0.00 & 0.59 \\
\hline 35 & Difficulty in remaining still and inactive & 0.59 & 0.13 & 0.03 & 0.16 & 0.57 \\
\hline
\end{tabular}

Factor loadings in bold indicate the factor on which item loaded

"Sensorimotor stereotypies" and "restricted behaviours" were significantly associated with severity of autistic symptoms assessed with the CARS and were more severely expressed in AD subjects compared to PDD-NOS subjects. However, while sensorimotor stereotypies appeared to be mainly associated with severe forms of AD, the restricted behaviours seemed to constitute a more complex dimension. In fact, "restricted behaviours" were related to autism but were also influenced by mental retardation and anxiety.

On the other hand, "reaction to change" was linked neither to severity of autistic symptoms nor to intellectual abilities. However, reactivity to change was greater in $\mathrm{AD}$ subjects than in PDD-NOS subjects. We can thus hypothesize that "reaction to change" is a specific marker of $\mathrm{AD}$, independent of the severity of symptoms or mental 
Table 3 Correlations between subscale scores and participants' characteristics

\begin{tabular}{|c|c|c|c|c|}
\hline Subscale & Age & DQ-IQ & CARS $^{\mathrm{a}}$ & Covi \\
\hline $\begin{array}{c}\text { F1—sensorimotor } \\
\text { stereotypies }\end{array}$ & $0.17 *$ & $-0.37 * * *$ & $0.47 * * *$ & 0.16 \\
\hline F2-reaction to change & 0.11 & -0.05 & -0.05 & 0.05 \\
\hline $\begin{array}{c}\text { F3-restricted } \\
\text { behaviours }\end{array}$ & -0.05 & $-0.26 * *$ & $0.31 * * *$ & $-0.25^{*}$ \\
\hline $\begin{array}{l}\text { F4-modulation } \\
\text { insufficiency }\end{array}$ & 0.14 & -0.14 & $0.29 * *$ & $0.40 * * *$ \\
\hline
\end{tabular}

${ }^{\text {a }}$ Partial correlation controlled for DQ-IQ

$* P \leq 0.05$; ** $P \leq 0.01$; *** $P \leq 0.001$

Table 4 Standard multiple regression analyses of variables explaining the subscale scores $(N=80)$

\begin{tabular}{llrlc}
\hline Subscale & Variable & \multicolumn{1}{l}{$\beta$} & SE $\beta$ & $t$ value \\
\hline F1-sensorimotor stereotypies & DQ-IQ & -0.05 & 0.10 & -0.53 \\
& CARS & 0.63 & 0.10 & $6.22^{* * *}$ \\
& Covi & 0.06 & 0.08 & 0.73 \\
F3-restricted behaviours & DQ-IQ & -0.29 & 0.11 & $-2.57^{*}$ \\
& CARS & 0.26 & 0.11 & $2.30^{*}$ \\
& Covi & -0.28 & 0.10 & $-2.87^{* *}$ \\
F4-modulation insufficiency & DQ-IQ & -0.04 & 0.12 & -0.37 \\
& CARS & 0.30 & 0.12 & $2.54^{*}$ \\
& Covi & 0.37 & 0.10 & $3.69 * * *$
\end{tabular}

$\beta=$ standardized regression coefficients, SE $\beta=$ standard error of $\beta$

* $P \leq 0.05$; ** $P \leq 0.01$; *** $P \leq 0.001$

retardation $[10,40]$. Previous studies have also proposed that these RRB are a feature of high-functioning autism and AS $[17,39]$. The sample in the present study did not comprise enough participants with good intellectual abilities to confirm this. Finally, it could be hypothesised that this dimension of the RRB scale might reflect an obsessive-compulsive trait that can be observed in some autistic subjects [46].

The fourth factor, "modulation insufficiency", is the only factor which did not differentiate AD from PDD-NOS, despite being linked to severity of autistic symptoms. The strong relationship between modulation insufficiency and anxiety suggests that such RRB might be related to anxiety disorders, which are frequently observed in ASD, rather than to autistic core symptoms [38]. Impulsiveness, agitation and emotional instability could also be explained by associated disorders such as ADHD or oppositional disorder.

\section{Anxiety and RRB}

Subjects with autism are known to have difficulties in communicating their emotional states; however, several studies have shown a high prevalence of anxiety in autistic disorder [19, 44]. Among the functions hypothesized for
RRB, they are thought to be coping strategies that allow autistic subjects to avoid or reduce the high level of anxiety resulting from a basic impairment of understanding the environment $[23,38,40]$. In the present study, two subscales were related to anxiety. We can thus hypothesize that the psychophysiological mechanisms of these two types of RRB are related to those of anxiety.

On the one hand, a high level of expression of "restricted behaviours" is associated with fewer manifestations of anxiety. Indeed, focusing on a restricted subject of interest or attraction to details of objects can correspond to avoiding strategies. By focusing on a restricted range of known and reassuring stimuli, the person avoids external stimuli that are difficult to integrate and that are stressful.

On the other hand, anxious participants had high levels of "modulation insufficiency". In fact, the different behavioural manifestations contained in this subscale seem to reflect difficulty with modulation of emotions. Therefore, subjects with a high score on this subscale may have major difficulties in adapting to a situation and controlling their feelings. The behaviours which make up the "modulation insufficiency" subscale can also be viewed as a means by which autistic subjects can exteriorize their internal states. Conversely, individuals with restricted behaviours, who tended to have severe autistic symptoms and associated mental retardation, probably had difficulties in communicating their anxiety.

\section{Comparison with previous findings on RRB}

"Sensorimotor stereotypies" (F1) and "reaction to change" (F2) are in accordance with the low/high-level model proposed by several authors [13, 39, 40], whereas "restricted behaviours" (F3) and "modulation insufficiency" (F4) constitute supplementary meaningful dimensions that could provide additional information for the understanding of RRB. "Restricted behaviours" include behaviours that were previously associated with different levels of ability; namely "restricted thoughts" considered as a high-level RRB and "interest in details of objects" placed in low-level RRB [4, 40]. Similarly, "modulation insufficiency" contains behaviours known to be associated with mental retardation (self-injury) [4, 23, 25] and less severe forms (stereotyped emotional manifestations). Except for self-injurious behaviours [23, 25], the behaviours included in the "modulation insufficiency" subscale have not been studied before as RRB. Interestingly, this dimension echoes the second factor of the BSE-R: a standardized scale which evaluates autistic behaviours [2].

Another scale assessing RRB has already been published: the RBS-R [6]. Both scales describe similar subtypes of RRB, i.e. reaction to change, sensorimotor activities and restricted behaviours [21], but there are 
differences between the two scales in terms of content and approach to behaviours. Although the RRB scale assesses behaviours that are not present in the RBS-R, the main difference is probably in the construction of their items. For the RBS-R each item corresponds to one precise behavioural manifestation (e.g. "insists on sitting in the same place", "insists on using a particular door", "hits self with body part", "hits self with object") whereas in the RRB scale each item includes different behavioural manifestations of the same hypothesized psychophysiological mechanism (e.g. "self-injurious behaviours", "rituals for daily living activities"). We believe that this complementary approach to RRB should provide an additional insight into these behaviours and improve the understanding of the underlying mechanisms of RRB.

\section{Strengths and limitations}

First, content validity allowed identification of the hypothesized multidimensional structure of the RRB scale and extraction of four statistically sound factors. Only two of the 35 items of the RRB scale failed to load on a single factor, and they were not included in the four subscales derived by PCA. However, this four-factor solution does not explain all the heterogeneity and complexity of RRB in ASD ( $43 \%$ of variance). It is, nevertheless, in accordance with previous factor analyses of RRB [21].

The sample was not completely representative of the ASD population, since $82 \%$ of the participants had mental retardation and the sample comprised only five subjects with AS. The proposed estimates of associated cognitive impairments in ASD populations currently range from 40 to $70 \%$ [3]. However, the study reported here included individuals from various centres that reflect the different residential settings offered in France. Further studies should be performed including a larger group of high-level autistic and Asperger individuals.

Finally, this variety of centres and the direct observation method used may have produced variations in the ratings of the RRB scale. In fact, the detailed glossary attached to the scale and the possibility of complementing the observation by information collected from relatives probably contributed to the good reliability and improved the accuracy of the evaluation.

\section{Conclusion}

The RRB scale provides a precise and reliable functional description of RRB over the whole autistic spectrum. It supports the multidimensionality of this field of autistic symptomatology. The description of different behavioural profiles should thus be valuable in research and clinical practice. It also supports the idea that dimensional analysis can be a more fruitful approach to the autistic spectrum than using diagnostic categories. Moreover, this new scale should help to characterize symptoms which are not specific to one psychiatric syndrome and which are an issue in differential diagnosis. Such an approach could also provide treatment indications and hypotheses on physiopathological mechanisms. For example, since RRB are observed in both ASD and in other psychiatric disorders, it would be interesting to evaluate RRB in subjects with mental retardation or OCD and then to compare their profiles to those of ASD individuals. Similarly, since RRB are known to be particularly persistent in autism, it would be interesting to study the potential differential evolution of the four types of RRB described by the scale and to assess their sensitivity to treatment.

Acknowledgments This research was supported by grants from the Foundation Orange. We thank the centres, raters and individuals that participated in the study: FAM les Maisonnées, Azay Le Rideau, France; Cabinet de Psychologie ESPAS, IDDEES, Paris, France; IME les Tilleuls, ADAPEI, Tours, France; Centre Hélio Marin, Saint Trojan les Bains, France; Hôpital de jour, Chevilly Larue, France; CIERA, Brest, France; CADIPA, Saint Egrève, France; CRA Basse Normandie, Caen, France; CRA Aquitaine, Bordeaux, France; Hôpital de jour Santos Dumont, Paris, France; CHS St-Jean-de-Dieu, Lyon, France; HUDERF, Bruxelles, Belgique; Centre de pédopsychiatrie, CHRU de Tours, France. We thank Rémy Magné for technical assistance.

\section{References}

1. American Psychiatric Association (2000) Diagnostic and statistical manual of mental disorders. American Psychiatric Press, Washington, DC

2. Barthélémy C, Roux S, Adrien JL, Hameury L, Guerin P, Garreau B, Fermanian J, Lelord G (1997) Validation of the revised behavior summarized evaluation scale. J Autism Dev Disord 27:139-153

3. Bebko JM, Schroeder JH, Weiss JA, Wells K, McFee K, Goldstein GM (2008) The face of autism research as reflected in the IMFAR looking glass. Res Autism Spectr Disord 2:385-394

4. Bishop SL, Richler J, Lord C (2006) Association between restricted and repetitive behaviors and nonverbal IQ in children with autism spectrum disorders. Child Neuropsychol 12:247-267

5. Bodfish JW, Symons F, Lewis M (1999) The repetitive behavior scale: a test manual. Western Carolina Center Research Reports, Morganton

6. Bodfish JW, Symons FJ, Parker DE, Lewis MH (2000) Varieties of repetitive behavior in autism: comparisons to mental retardation. J Autism Dev Disord 30:237-243

7. Bourreau Y, Roux S, Gomot M, Barthélémy C (in press) Repetitive and Restricted Behaviours (RRB) in autism: clinical evaluation. L'Encéphale

8. Brunet O, Lézine I (2001) Brunet-Lezine psychomotor development test. ECPA, Paris

9. Campbell M (1985) Timed stereotypies rating scale. Psychopharmacol Bull 21:1082

10. Carcani-Rathwell I, Rabe-Hasketh S, Santosh PJ (2006) Repetitive and stereotyped behaviours in pervasive developmental disorders. J Child Psychol Psychiatry 47:573-581 
11. Cicchetti DV, Sparrow SA (1981) Developing criteria for establishing interrater reliability of specific items: applications to assessment of adaptive behavior. Am J Ment Defic 86:127-137

12. Covi L, Lipman RS, McNair DM, Czerlinsky T (1979) Symptomatic volunteers in multicenter drug trials. Prog Neuropsychopharmacol 3:521-533

13. Cuccaro ML, Shao Y, Grubber J, Slifer M, Wolpert CM, Donnelly SL, Abramson RK, Ravan SA, Wright HH, DeLong GR, Pericak-Vance MA (2003) Factor analysis of restricted and repetitive behaviors in autism using the autism diagnostic interview-R. Child Psychiatry Hum Dev 34:3-17

14. Fermanian J (1984) Measurement of agreement between 2 judges. Qualitative cases. Rev Epidemiol Santé Publique 32:140-147

15. Fermanian J (1984) Measuring agreement between 2 observers: a quantitative case. Rev Epidemiol Santé Publique 32:408-413

16. Gabriels RL, Cuccaro ML, Hill DE, Ivers BJ, Goldson E (2005) Repetitive behaviors in autism: relationships with associated clinical features. Res Dev Disabil 26:169-181

17. Georgiades S, Szatmari P, Zwaigenbaum L, Duku E, Bryson S, Roberts W, Goldberg J, Mahoney W (2007) Structure of the autism symptom phenotype: a proposed multidimensional model. J Am Acad Child Adolesc Psychiatry 46:188-196

18. Howlin P, Goode S, Hutton J, Rutter M (2004) Adult outcome for children with autism. J Child Psychol Psychiatry 45:212-229

19. Kim JA, Szatmari P, Bryson SE, Streiner DL, Wilson FJ (2000) The prevalence of anxiety and mood problems among children with autism and Asperger syndrome. Autism 4:117-132

20. Krug DA, Arick J, Almond P (1980) Behavior checklist for identifying severely handicapped individuals with high levels of autistic behavior. J Child Psychol Psychiatry 21:221-229

21. Lam KS, Aman MG (2007) The repetitive behavior scale-revised: independent validation in individuals with autism spectrum disorders. J Autism Dev Disord 37:855-866

22. Lecrubier Y (1992) Echelle de gravité de l'anxiété de Covi. In: Guelfi JD (ed) L'évaluation clinique standardisée en psychiatrie. Pierre Favre, Paris, pp 303-307

23. Lewis MH, Bodfish JW (1998) Repetitive behavior disorders in autism. Ment Retard Dev Disabil Res Rev 4:80-89

24. Lord C, Rutter M, Le Couteur A (1994) Autism diagnostic interview-revised: a revised version of a diagnostic interview for caregivers of individuals with possible pervasive developmental disorders. J Autism Dev Disord 24:659-685

25. Militerni R, Bravaccio C, Falco C, Fico C, Palermo MT (2002) Repetitive behaviors in autistic disorder. Eur Child Adolesc Psychiatry 11:210-218

26. Murphy GH, Beadle-Brown J, Wing L, Gould J, Shah A, Holmes $\mathrm{N}$ (2005) Chronicity of challenging behaviours in people with severe intellectual disabilities and/or autism: a total population sample. J Autism Dev Disord 35:405-418

27. Papageorgiou V, Georgiades S, Mavreas V (2007) Brief report: cross-cultural evidence for the heterogeneity of the restricted, repetitive behaviours and interests domain of autism: a greek study. J Autism Dev Disord 38:558-561

28. Perron-Borelli M (2005) Differential scales of intellectual efficiencies: revised. ECPA, Paris

29. Perry A, Condillac RA, Freeman NL, Dunn-Geier J, Belair J (2005) Multi-site study of the Childhood Autism Rating Scale
(CARS) in five clinical groups of young children. J Autism Dev Disord 35:625-634

30. Richler J, Bishop SL, Kleinke JR, Lord C (2007) Restricted and repetitive behaviors in young children with autism spectrum disorders. J Autism Dev Disord 37:73-85

31. Rickels K, Csanalosi I, Greisman P, Mirman MJ, Morris RJ, Weise CC, Weiss G (1980) Ketazolam and Diazepam in anxiety: a controlled study. J Clin Pharmacol 20:581-589

32. Roid GH, Miller LJ (1997) Leiter international performance scale: revised. Stoelting Co., Woodale

33. Rojahn J, Matson JL, Lott D, Esbensen AJ, Smalls Y (2001) The Behavior Problems Inventory: an instrument for the assessment of self-injury, stereotyped behavior, and aggression/destruction in individuals with developmental disabilities. J Autism Dev Disord 31:577-588

34. Rojahn J, Tasse MJ, Sturmey P (1997) The stereotyped behavior scale for adolescents and adults with mental retardation. Am J Ment Retard 102:137-146

35. Schopler E (1994) Psycho educative profile: revised, translated. De Boeck, Bruxelles

36. Schopler E, Reichler RJ, DeVellis RF, Daly K (1980) Toward objective classification of childhood autism: Childhood Autism Rating Scale (CARS). J Autism Dev Disord 10:91-103

37. Seltzer MM, Krauss MW, Shattuck PT, Orsmond G, Swe A, Lord C (2003) The symptoms of autism spectrum disorders in adolescence and adulthood. J Autism Dev Disord 33:565-581

38. Sukhodolsky DG, Scahill L, Gadow KD, Arnold LE, Aman MG, McDougle CJ, McCracken JT, Tierney E, Williams White S, Lecavalier L, Vitiello B (2007) Parent-rated anxiety symptoms in children with pervasive developmental disorders: frequency and association with core Autism symptoms and cognitive functioning. J Abnorm Child Psychol 36:117-128

39. Szatmari P, Georgiades S, Bryson S, Zwaigenbaum L, Roberts W, Mahoney W, Goldberg J, Tuff L (2006) Investigating the structure of the restricted, repetitive behaviours and interests domain of autism. J Child Psychol Psychiatry 47:582-590

40. Turner M (1999) Annotation: repetitive behaviour in autism: a review of psychological research. J Child Psychol Psychiatry 40:839-849

41. Wechsler D (1996) Wechsler intelligence scale for children (WISC III) - translated. ECPA, Paris

42. Wechsler D (2005) Wechsler intelligence scale for children (WISC IV)-translated. ECPA, Paris

43. Wechsler D (2002) Wechsler preschool and primary scale of intelligence (WPPSI III) - translated. ECPA, Paris

44. Weisbrot DM, Gadow KD, DeVincent CJ, Pomeroy J (2005) The presentation of anxiety in children with pervasive developmental disorders. J Child Adolesc Psychopharmacol 15:477-496

45. World Health Organization (1997) Tenth revision of the International Classification of Diseases and Related Health Problems (ICD-10). WHO, Geneva

46. Zandt F, Prior M, Kyrios M (2007) Repetitive behaviour in children with high functioning autism and obsessive compulsive disorder. J Autism Dev Disord 37:251-259

47. Zazzo R, Gilly M, Verba-Rad M (1985) New metric scale of intelligence (NEMI). EAP, Paris 